\title{
Guía para el Trabajo Clínico en Odontología Durante Pandemia por SARS-CoV-2, en el Hospital Clínico Dra. Eloísa Díaz
}

\author{
Guide for Clinical Work in Dentistry During SARS-CoV-2 \\ Pandemic, at the Hospital Clínico Dr. Eloísa Díaz
}

Inés Aylwin Miranda; Carla Richaud Hiza \& Yasna Moreno Yañez

AYLWIN, M. I.; RICHAUD,H. C. \& MORENO, Y. Y. Guía para el trabajo clínico en odontología durante pandemia por SARSCoV-2, en el Hospital Clínico Dra. Eloísa Díaz. Int. J. Odontostomat., 15(1):51-58, 2021.

RESUMEN: Un nuevo brote de coronavirus anunciado a principios de este año ha sido asociado a la renombrada enfermedad de "COVID-19" que se ha convertido en la más severa pandemia de este siglo. Tal como lo anunció en marzo del 2020, la Organización Mundial de la Salud (OMS), corresponde a una emergencia de salud pública que repercute fuertemente a nivel internacional (Meng et al., 2020). El siguiente artículo busca generar un documento que registre las orientaciones y medidas de prevención de COVID-19 en la odontología basado en la evidencia. Para esto cobra gran importancia las medidas de equipo de protección personal (EPP) que se deben utilizar. Aunque la evidencia es variada y se dice que las medidas estándares no son suficientes para prevenir la diseminación cuando se trabaja con aerosoles (Facultad de Odontología de la Universidad de Chile, 2020), es necesario, de forma paralela que avanza la propagación, ir generando consciencia con medidas muy cuidosas y claras en cada espacio sanitario. Por lo expuesto anteriormente y en consideración el contexto nacional, es necesario definir las medidas standard y específicas para la atención odontológica, en este caso dentro de la Unidad Dental del Hospital Clínico Dr. Eloísa Díaz (HLF).

PALABRAS CLAVE: COVID-19; odontología; protocolo clínico.

\section{INTRODUCCIÓN}

Según las estadísticas mundiales de salud publicadas al día 2 de agosto de 2020 , existen 17.660 .523 casos y 680.894 muertes (World Health Organization, 2020). A nivel nacional de acuerdo con el reporte epidemiológico del Ministerio de Salud (MINSAL) se registra cerca de 350.131 casos confirmados acumulados y 9.608 fallecidos (Ministerio de Salud, 2020a,b).

Si bien la información sobre la propagación del virus está en constante actualización, Peng et al. (2020) afirman que "las rutas comunes de transmisión del virus incluyen la transmisión directa, es decir, tos, estornudos y transmisión por inhalación de gotitas y la transmisión por contacto (contacto con las membranas mucosas orales, nasales y oculares)". Además, añade que la exposición ocular puede ser una manera muy efectiva para que el virus ingrese al cuerpo.
También es posible contraer el virus de modo indirecto al tocar una superficie u objeto contaminado y luego tocarse la boca, la nariz o los ojos lo que aumenta más aún el riesgo de infectarse (Subsecretaría de Salud Pública, 2020a,b).

Es notable lo que To et al. (2020) informaron en su estudio donde mediante medios de cultivos de saliva de pacientes infectados, demostró que en la mayoría de ellos se detectó altos títulos de carga viral viva que pueden permitir la transmisión. Incluso en algunos pacientes, el coronavirus se detectó solo en la saliva, pero no en el hisopado nasofaríngeo. Esto indica además que el Covid-19 puede también transmitirse a través de la saliva directa o indirectamente incluso entre pacientes sin tos u otros síntomas respiratorios (Peng et al.). 
Lo anterior sumado a la evidencia de que el virus puede estar en el aire a través de los aerosoles formados durante procedimientos médicos y dentales como, por ejemplo, el instrumental rotatorio, refuerzan la idea de que en el entorno del trabajo odontológico se genera inevitablemente un alto riesgo de infección de Covid-19 (Peng et al., 2020). Es decir, la especificidad de los procedimientos, la estrecha distancia necesaria para la atención de pacientes y la frecuente exposición a la saliva y otros fluidos nos expone a diario a este patógeno promoviendo al personal de salud bucal en un complejo escenario con mayor riesgo de contraer la enfermedad y en consecuencia continuar con la cadena de propagación de ésta.

Por lo expuesto anteriormente y en consideración al contexto nacional, es necesario definir las medidas standard y específicas para la atención odontológica, en este caso dentro de la Unidad Dental del HLF.

Medidas de precaución estándar (Subsecretaria de Salud Pública, 2020a,b). Es fundamental que todo el personal de salud esté en particular preocupación por mantener la correcta ejecución y totalidad de las medidas de precaución:

- Higiene de manos (Fig. 1).

- Uso de guantes.

- Protección facial.

- Uso de delantal.

- Prevención de pinchazos y accidentes cortopunzantes.

- Higiene respiratoria y buenos hábitos al toser o estornudar.

- Manejo del ambiente, equipos, desechos y ropa del paciente.

Sala de espera (Subsecretaria de Redes Asistenciales, 2020; Araya, 2020; Ministerio de Salud, $2020 a, b)$. Con el objetivo de disminuir el riesgo de contagio mientras el paciente espera ser atendido y facilitar la constante desinfección de superficies que requiere la sala a continuación se entregan recomendaciones en cuanto a las medidas a aplicar en la sala de espera previo a la llegada de los pacientes e indicaciones que se les debe otorgar a éstos.

a) Etapas previas a la atención:

- Retirar revistas, folletos, juguetes, dispensadores de alimentos/agua y elementos no necesarios de las salas de espera.
- Organizar el mobiliario con la mínima cantidad de sillas posibles y separadas, para respetar el distanciamiento de 1.5 metros idealmente.

- Asegurar el distanciamiento social en la sala de espera mediante el uso de señaléticas en el suelo, sillas y otros mobiliarios.

- Desinfección de superficies en áreas de ascensores, recepción, sala de espera y especialmente de las áreas clínicas, con soluciones de hipoclorito de sodio o amonio cuaternario.

- Solicitar a pacientes puntualidad y no asistir acompañados, de lo contrario el acompañante debe esperar fuera del hospital (Salvo excepciones, por ejemplo: pacientes pediátricos).

- Instalar infografías de recomendaciones sanitarias en paredes de un material que permita su desinfección: uso de mascarillas, lavado de manos constante y distanciamiento social.

b) En relación a los pacientes

- Asistir con mascarilla quirúrgica, de no ser posible mascarilla de paso y mantener el distanciamiento social recomendado.

- Mínima cantidad de pacientes posibles en la sala de espera, para ello espaciar las citas de atención. Se sugiere 1 hora de atención por cada paciente para otorgar tiempo de desinfección y ventilación.

- Tiempo de espera deben ser cortos, se recomienda un máximo de 20 min.

c) Sala de espera

- Debe ser ventilada, de no ser posible, se recomienda aumentar la distancia entre pacientes. Por ejemplo, delimitar cuatro asientos de distancia entre pacientes.

- Debe tener acceso a lavado de manos para los pacientes y sus acompañantes con dispensadores de solución en base a alcohol gel.

- El lugar de recepción de pacientes deberá contar con elementos de protección como pantallas de protección o vidrio de separación. Si no es posible, indicar el uso de mascarillas o escudo facial.

Clasificación de pacientes (Subsecretaria de Redes Asistenciales; Martínez-Camus \& YévenesHuaiquinao, 2020).

a) Caso no sospechoso de infección SARS-CoV-2.

- Paciente asintomático o con diagnóstico negativo confirmado (24 horas previo a la consulta). 


\section{¿Cómo lavarse las manos?}

(4) Duración de todo el procedimiento: 40-60 segundos
0

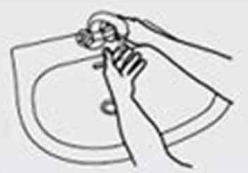

Mojese las manos con agua;
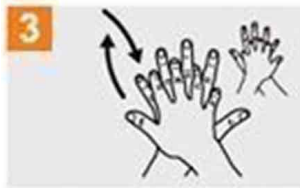

Frótese la palma de la mano derech contra el dorso de la mano izquierda entrelazando los dedos y viceversa;

6

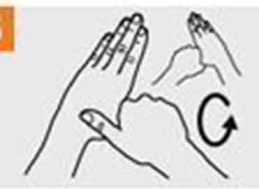

Froteso con un movimiento de rotación el pulgar izquierdo. rotación el pulgar izquierdo, mano derocha y viceversa;

9

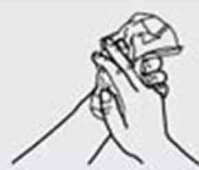

Sequese con una toalla desechable:

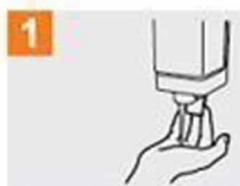

Deposite en la palma de la mano una cantidad de jabón suficiente para cubrir todas las superficies de las manos;
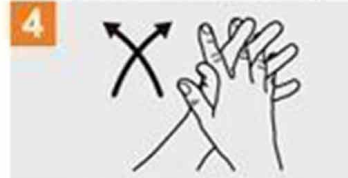

Frótoso las palmas do las manos entre si, con los dedos entrelazados;

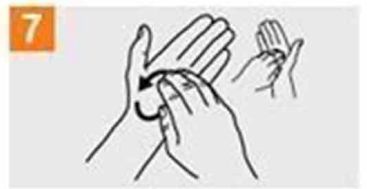

Frótese la punta de los dedos de la mano derecha contra la palma de la mano izquierda, haciendo un movimiento de rotación y vicoversa;
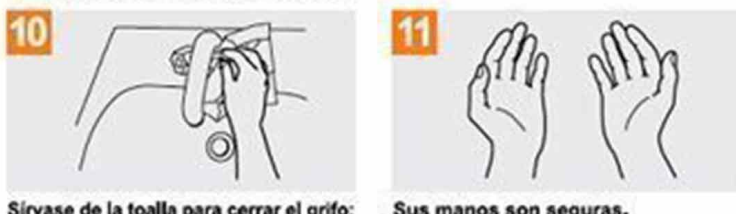

Sus manos son seguras.

Seguridad del Paciente

SAVE LIVES

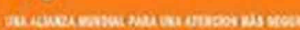

Olean Your Hands

(4.) Organización

Fig. 1. Cómo lavarse las manos (World Health Organization, 2009).

- Puede ser atendido de manera inmediata.

- Realizar tratamientos de urgencia odontológica y electivos minimizando procedimientos que generen aerosoles (PGA), en estos casos impostergables de PGA, utilizar goma dique.

- En casos de paciente con diagnóstico negativo pero perteneciente al grupo de alto riesgo de morbilidad/ mortalidad realizar solo tratamientos de urgencias o emergencia odontológica. Evaluar exodoncias vs endodoncias complejas según criterio clínico.

b) Caso sospechosos o probables de infección SARSCoV-2, no confirmado:

- Pacientes que presentan al menos dos síntomas de la enfermedad o cualquier persona con infección res- piratoria aguda que requiera hospitalización.

- Casos probables: Personas que han sido expuestas a un contacto estrecho de un paciente confirmado con Covid-19 y que presenta al menos 1 síntoma.

- Posponer toda atención electiva. Solo tratar urgencias o emergencias minimizando PGA. Podrá realizarse tratamiento electivo una vez transcurridos 15 días después de confirmar que son COVID (-), o 30 días después del periodo de cuarentena en caso de ser confirmados COVID (+).

c) Caso confirmado de COVID19:

- Caso sospechoso en que el examen PCR para Covid-19 resultó positivo.

- Diferir todo tratamiento no urgente.

- Si corresponde a una emergencia impostergable, debe ser coordinada con el médico, y realizarse en una sala AIIR o en salas de presión negativa con un mínimo de 12 cambios de aire por hora o al menos $160 \mathrm{~L} / \mathrm{s}$ por paciente.

- Manejo multidisciplinario. Se sugiere ser derivado a emergencia médica para atención.

d) Paciente COVID-19 recuperado:

- Caso recuperado, no contagiado de COVID-19. No existe consenso de cuándo un paciente que fue diagnosticado con la enfermedad puede interrumpir el aislamiento en el hogar. Sin embargo, el paciente es considerado portador del virus hasta después de 30 días de confirmar su recuperación con una prueba de laboratorio. Se atenderá si el paciente ha estado asintomático desde 14 a 30 días posterior al inicio de sus síntomas o fue diagnosticado.

- Realizar tratamientos de urgencia odontológica y tratamientos electivos minimizando PGA, optando por aislación relativa evaluando el riesgo según cada caso y criterio clínico. 
Tabla I. Cuestionario TRIAGE (Hospital Clínico Dra. Eloísa Díaz I., 2020).
1) ¿Tiene fiebre o la ha tenido en los últimos 14 días (temperatu ra $>37,8^{\circ} \mathrm{C}$ )?
2) ¿Ha tenido tos o cualquier otro signo respiratorio en los últimos 14 días?
3) ¿Ha tenido o tiene diarrea u otras molestias digestivas en los últimos 14 días?
4) ¿Tiene o ha tenido sensación de mucho cansancio o malestar en los últimos 14 días?
5) ¿Ha notado una pérdida del sentido del gusto o del olfato en los últimos 14 días?
6) ¿Ha estado en contacto o conviviendo con alguna persona sospechosa o confirmada de coronavirus?
7) ¿Fue diagnosticado con COVID-19?
8) En caso de haber sido diagnosticado con COVID-19, ¿sigue usted en cuarentena?

Preparación del paciente (Subsecretaria de Salud Pública, 2020a,b; Subsecretaria de Redes Asistenciales; Bermúdez-Jiménez et al., 2020). Se requiere organizar las etapas de atención, para otorgar eficacia y seguridad tanto al paciente como al personal del Hospital. Todo paciente se considera como potencial sospechoso de SARS-CoV-2 hasta que se demuestre lo contrario.

\section{a) Previo a la atención}

- Evaluación de la necesidad de atención: Realizar triage telefónico o vía correo electrónico, de no ser posible, implementar el triage presencial (Tabla I). Evitar asistencias innecesarias y limitarse a la presencia del paciente y un acompañante máximo, solo en caso de ser necesario.

- Categorización tipo de atención: Evaluar tipo de tratamiento dental requerido, en conjunto a antecedentes del paciente, para determinar riesgos y beneficios de ser atendido.

- Evaluar la necesidad y urgencia del tratamiento.

- Clasificar al paciente. Según esto, dar o no hora de atención.

b) En la sala de espera

- Solicitar lavado de manos con agua y jabón en el baño disponible para pacientes.

- Confirmar y realizar nuevamente triage escrito en la recepción.

- Tomar y registrar temperatura del paciente.

- Pedirle que firme consentimiento escrito donde se consigne su condición de salud y el tipo de la atención odontológica en contexto de Pandemia SARSCoV-2, que recibirá.

c) Previo ingreso al box el paciente debe:

- Retirar elementos accesorios como aros, collares, pulseras, reloj (Realizarlo en sala de espera, no justo antes de ser atendido).
- Uso obligatorio de mascarilla quirúrgica idealmente o de paso.

- Limpiar pies con paño desinfectante dispuesto en la Unidad, con solución de hipoclorito o amonio cuaternario y luego secarlos en la alfombra puesta a continuación.

- Asistir al control odontológico con la higiene oral ya realizada previamente en su hogar

- Segundo lavado de manos o desinfección con alcohol gel

d) En el box de atención:

- Considerar algún lugar establecido para que el paciente pueda dejar sus pertenencias. Se aconseja disponer de una bolsa plástica grande para que el paciente pueda colocar sus pertenencias y así mantenerlas cerca de él.

- Solicitar a paciente tercer lavado de manos, o desinfección con alcohol gel

- Indicar que no toque nada y tomar asiento en sillón dental

- Disponer de enjuague bucal de Gluconato de clorhexidina $0,12 \%$ o povidona yodada al $0,2 \%$ durante 30 segundos.

Preparación del personal auxiliar y profesional (Ministerio de Salud, 2020a,b; Alharbi et al., 2020).

- Todo el equipo de salud debe estar con los EPP instalados cuando el paciente ingrese para la atención odontológica.

- El uso de mascarilla quirúrgica es obligatorio en todo momento.

- Los trajes clínicos y zapatos deben ser distintos a la vestimenta de circulación fuera del Hospital.

- Técnico a cargo de realizar etapa preparatoria de ingreso y triage del paciente, también debe utilizar todos los EPP recomendados.

Previo a la colocación de los EPP, el profesional debe utilizar el traje clínico con zapatos cerrados, 
retirarse los accesorios (anillos, aros, collares y reloj), amarrarse el cabello y/o asegurar que no se le desplace sobre la cara y finalmente debe siempre lavarse las manos antes y después de la atención (Fig. 1) (Ministerio de Salud, 2020a,b; Colegio Médico de Chile \& Sociedad Chilena de Infectología, 2020). Para eso es importante que se planifique la sesión dental con anticipación.

Sobre el traje clínico el profesional de la salud deberá colocarse los siguientes elementos de protección personal (Organización Mundial de la Salud, 2020):

- Mascarilla quirúrgica.

- Máscara facial: debe desinfectarse entre pacientes

- Gafas protectoras: debe desinfectarse entre pacientes.

- Gorro.

- Guantes desechables.

- Cubre calzado desechable.

- Sugerencia: utilizar doble gorro y guante.

En el caso de atenciones con generación de aerosoles se deberá utilizar las medidas de EPP señaladas anteriormente $y$, además:

- Delantal impermeable de manga larga desechable - Mascarilla N95 o máscaras FFP3.

Preparación del box clínico. El box dental debe cumplir con las siguientes medidas (Subsecretaría de Redes Asistenciales; Asociación Latinoamericana de Odontopediatría, 2020; Facultad de Odontología de la Universidad de Chile, 2020).

- Cumplir idealmente con medidas de ventilación (sistema de presión negativa o presencia de ventana).

- Procurar mantener la temperatura del box lo más alta posible y seco, evitar encender aire acondicionado.

- Desinfección de superficies con alcohol $70 \%$, cloro $0,1 \%$ o amonio cuaternario.

- Tener a la vista solamente el material indispensable que vaya a usarse dejando todo el demás material embolsado en las cajoneras.

- Colocar film plástico sobre aquellas superficies con mayor contacto durante la práctica (botones del equipo, foco del sillón dental, bandeja del sillón dental, etc.), el cual deberá ser cambiado entre pacientes.

- Todo aparato que pueda estar en contacto con la boca del paciente (por ejemplo, lámpara de fotocurado, espejos intraorales, turbina, etc.) debe estar protegida con funda descartable.
Consideraciones durante la atención dental (Sociedad Chilena de Cirugía y Traumatología Bucal y Maxilofacial, 2020; Hospital Clínico Dra. Eloísa Díaz I., 2020; Subsecretaría de Salud Pública, 2020a,b; Ather et al., 2020; Meng et al., 2020).

- Realizar lavado de manos con agua y con jabón.

- Sólo se permitirá un máximo de 4 personas por box, dentro de las cuales se incluye: Dentista, Asistente, Paciente y Acompañante o Alumno/a en práctica.

- El equipo de salud debe estar con los EPP instalados cuando el paciente ingrese para la atención odontológica, y cuando el paciente ingrese al lugar de atención, se debe lavar las manos o higienizar con alcohol gel, y luego tomar contacto con el sillón dental.

- Entregar al paciente y su acompañante elementos de protección ocular y barreras físicas como pecheras plásticas lavables o desechables.

- Realizar atención a cuatro manos y utilizar goma dique cada vez que sea posible.

- Durante la atención no manipular ningún dispositivo electrónico.

- Se deberá trabajar con la puerta del box cerrada.

- Se deberá cubrir con funda descartable la manguera del aspirador y eyector, la cual debe ser cambiada o desinfectada entre cada atención.

- Evitar el uso de jeringa triple, de preferencia secar con gasa. En caso usarla se deberá utilizar una funda descartable en la manguera de esta.

- Evitar uso de ultrasonido, scaler y turbina para disminuir formación de aerosoles.

- Si el uso de material rotatorio es indispensable, solamente se recomienda usarlo en caso de disponer de aspiración de alta potencia, separadores labiales con aspiración de aerosoles y de poder recurrir al aislamiento con dique de goma. De lo contrario NO se recomienda usar material rotatorio.

- En tratamientos donde no sea posible aislar preferir técnicas como ART o instrumentación manual.

- Se sugiere solicitar exámenes imagenológicos extraorales en vez de intraorales con objetivo de evitar el contacto con la saliva y estimular el reflejo de tos del paciente. En caso de requerir su utilización envolver el sensor con film plástico o envoltorios desechables. Luego de tomar la radiografía, retirar el film del sensor y desinfectarlo con alcohol en gel o líquido.

Consideraciones posteriores a la atención dental. Una vez terminada la atención con el paciente se deberán seguir las siguientes medidas: (Vargas-Buratovic et al., 2020; Subsecretaría de Redes Asistenciales; 
Hospital Clínico Dra. Eloísa Díaz I.; Colegio Médico de Chile \& Sociedad Chilena de Infectología; Ministerio de Salud, 2020a,b).

- Implementar el lavado de manos con agua y jabón de $\mathrm{CHX}$ por parte del personal odontológico y pacientes.

- Desechar los elementos de protección personal no reutilizables. Se deben extremar los cuidados a la hora de retirarse los EPP con el objetivo de no contaminarse (Figs. 2 y 3 ).

- Todo el material desechable utilizado debe ir a contenedores especiales para desechos biológicos.

- Desinfección del material de protección ocular y máscara facial.

- Todo el instrumental será llevado al área de desinfección y esterilización, portando siempre los EPP.

- Si se utilizó material rotatorio se debe desinfectar con alcohol $70 \%$, para posteriormente ser esterilizado.

- Limpiar y desinfectar superficies de uso frecuente con solución de hipoclorito de sodio al $0.1 \%$ o amonio cuaternario.

- Con la misma solución de hipoclorito de sodio rociar el ambiente.

- Se recomienda ventilación de la sala después de la atención. En caso de haber realizado procedimiento con generación de aerosoles, se deberá dejar ventilar por 30 minutos. En caso de procedimientos sin generación de aerosoles bastará con 15-30 minutos.

El uniforme clínico debe ser transportado en bolsa plástica desechable, queda estrictamente prohibido salir de la consulta con uniforme clínico. Al llegar a casa, el personal de salud debe: (Asociación Latinoamericana de Odontopediatría; Subsecretaría Redes Asistenciales).

- Retirarse el calzado y desinfectarlo

- Retirarse las ropas y lavarlas separadas del resto de las ropas de otros residentes del hogar

- Ducharse inmediatamente

\section{CONCLUSIONES}

Con el objetivo de prevenir la transmisión de los agentes microbianos, evitar la contaminación y generar un ambiente seguro en las unidades dentales, se deben aplicar las medidas de precaución y las medidas señaladas en la revisión, de manera universal, se trate o no de pacientes contagiados. Es funda-

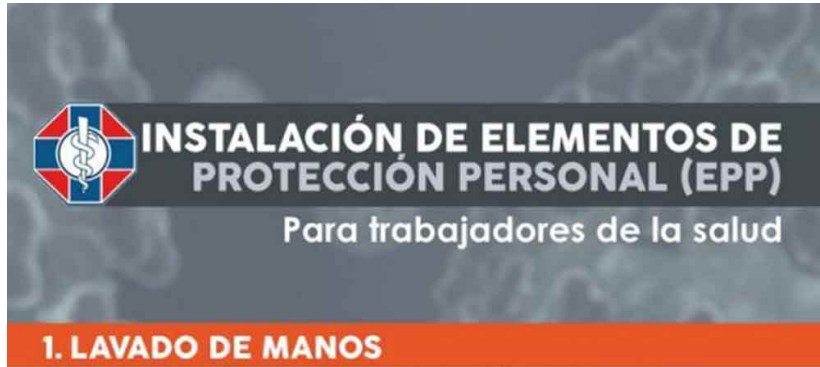

Siempre, antes de la instalación de las medidas de EPP se debe realizar lavado de manos clínico con agua y jabón.

2. INSTALACIÓN DE PECHERA

Instalar pechera con mangas de forma individual.

3. INSTALACIÓN DE MASCARILLA Instalar mascarilla quirúrgica. Instalar mascarilla N95 para procedimientos que generen aerosoles.

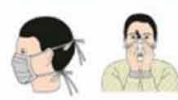

\section{INSTALACIÓN DE PROTECCIÓN OCULAR}

Instalar protección ocular: Gafas de seguridad tipo antiparras. Visor, con protección facial.

\section{INSTALACIÓN DE GUANTES}

Instalación guantes de procedimiento (por encima de los puños de la pechera).

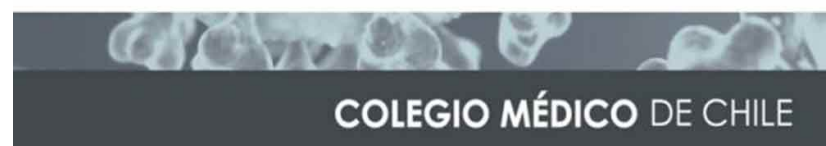

Fig. 2. Secuencia de colocación y retiro de EPP (Colegio Médico de Chile \& Sociedad Chilena de Infectología, 2020).

mental realizar previamente un triage para evaluar el tipo de atención y categorización del paciente. Debe usarse el material rotatorio solo en casos indispensables. Para pacientes con sintomatológia o COVID-19 confirmados con emergencia impostergable, su atención debe ser coordinada con manejo médico, y realizarse en una sala AIIR o en salas de presión negativa con un mínimo de 12 cambios de aire por hora.

AYLWIN, M. I.; RICHAUD,H. C. \& MORENO, Y. Y. Guide for clinical work in dentistry during SARS-CoV-2 pandemic, at the Hospital Clínico Dr. Eloísa Díaz. Int. J. Odontostomat., 15(1):51-58, 2021. 


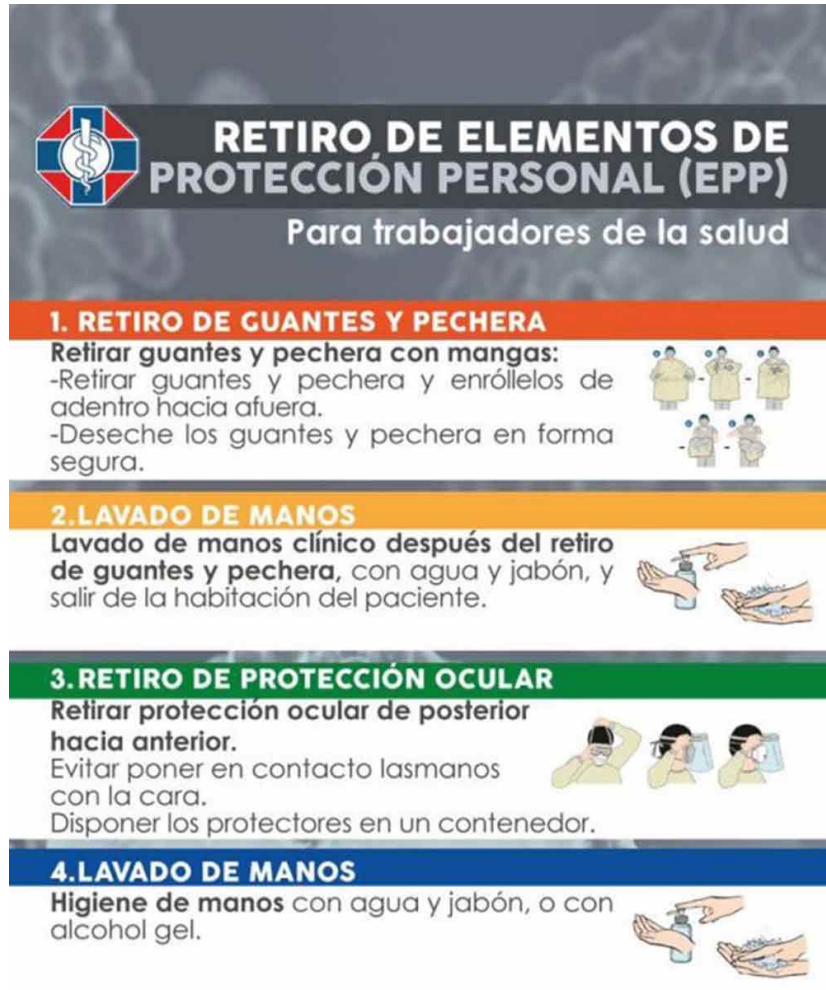

5.RETIRO DE MASCARILLAS

Retirar mascarilla desde atrás y desechar en forma segura.

\section{LAVADO DE MANOS}

Lavado de manos clínico con agua y jabón.

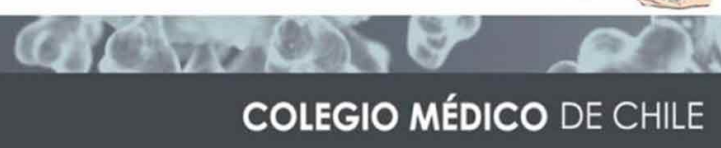

Fig. 3. Secuencia de colocación y retiro de EPP (Colegio Médico de Chile \& Sociedad Chilena de Infectología, 2020).

ABSTRACT: A new coronavirus outbreak announced earlier this year has been associated with the renowned "COVID-19" disease that has become the most severe pandemic of this century. As announced in March 2020, by the World Health Organization (WHO) this corresponds to a public health emergency that has strong repercussions at the international level (Meng et al., 2020). The following article seeks to generate a document that records the guidance and prevention measures of COVID-19 in dentistry based on the evidence. For this, the personal protective equipment (PPE) measures that must be used are of great importance. Although the evidence is varied and it is said that standard measures are not sufficient to prevent dissemination when working with aerosols (Facultad de Odontología de la Universidad de Chile, 2020), it is necessary, as the virus spreads to generate awareness for extremely clear and careful measures for sanitary space in this context. Due to the above and in consideration of the national context, it is necessary to define the standard and specific measures for dental care and in this case within the Dental Unit of the Dr. Eloísa Díaz Clinical Hospital (HLF). protocol.

KEY WORDS: COVID-19, dentistry, clinical

\section{REFERENCIAS BIBLIOGRÁFICAS}

Alharbi, A.; Alharbi, S. \& Alqaidi, S. Guidelines for dental care provision during the COVID-19 pandemic. Saudi Dent. J., 32(4):181-6, 2020.

Araya, C. Considerations for emergency dental care and preventive measures for COVID-19 (SARS-CoV 2). Int. J. Odontostomat., 14(3):268-70, 2020.

Asociación Latinoamericana de Odontopediatría. Ruta de Atención para Procedimientos de Odontología Pediátrica durante la Etapa de Confinamiento o Cuarentena de la Pandemia COVID-19. Rev. Odontopediatría Latinoam., 10(2), 2020. Disponible en: https://www.revistaodontopediatria.org/ediciones/2020/2/art-1/

Ather, A.; Patel, B.; Ruparel, N. B.; Diogenes, A. \& Hargreaves, K. M. Coronavirus Disease 19 (COVID-19): implications for clinical dental care. J. Endod., 46(5):584-95, 2020.

Bermúdez-Jiménez, C.; Gaitán-Fonseca, C. \& Aguilera-Galaviz, L. Manejo del paciente en atención odontológica y bioseguridad del personal durante el brote de coronavirus SARS-CoV-2 (COVID-19). Rev. A. D. M., 77(2):88-95, 2020.

Colegio Médico de Chile \& Sociedad Chilena de Infectología. Infecciones asociadas a la atención de salud. Recomendaciones uso de elementos de protección personal (EPP): Para trabajadores de la salud. Rev. Chil. Infectol., 37(2):106-10, 2020.

Facultad de Odontología de la Universidad de Chile. Recomendaciones para la Atención Odontológica frente a la pandemia por COVID19/SARS CoV-2. Santiago de Chile, Facultad de Odontología de la Universidad de Chile, 2020. Disponible en: http://web.uchile.cl/ facultades/odontologia/recomendacionesCOVID19/2/

Hospital Clínico Dra. Eloísa Díaz I. Protocolo de Atención de Pacientes en Servicio Dental durante Pandemia por SARS-CoV-2 en Hospital Clínico Dra. Eloísa Díaz I. La Florida, Hospital Clínico Dra. Eloísa Díaz I., 2020.

Martínez-Camus, D. C. \& Yévenes-Huaiquinao, S. R. Dental care during COVID-19 Pandemic. Int. J. Odontostomat., 14(3):28895, 2020.

Meng, L.; Hua, F. \& Bian, Z. Coronavirus Disease 2019 (COVID19): emerging and future challenges for dental and oral medicine. J. Dent. Res., 99(5):481-7, 2020.

Ministerio de Salud. Casos Confirmados en Chile COVID-19. Santiago de Chile, Ministerio de Salud, Gobierno de Chile, 2020b. Disponible en: https://www.minsal.cl/nuevo-coronavirus-2019ncov/casos-confirmados-en-chile-covid-19/

Ministerio de Salud. Protocolo de Limpieza y Desinfección de Ambientes - COVID-19. Plan de Acción Coronavirus COVID-19. Santiago de Chile, Ministerio de Salud, Gobierno de Chile, 2020a. Disponible en: http://www.colegiomedico.cl/wp-content/uploads/ 2020/03/PROTOCOLO-DE-LIMPIEZA-Y-DESINFECCIÓN-DEAMBIENTES-COVID-19.pdf

Organización Mundial de la Salud. Equipo de Protección Personal. Ginebra, Organización Mundial de la Salud, 2020. Available from: https://www. who.int/csr/resources/publications/eppoms.pdf?ua=1 
Peng, X.; Xu, X.; Li, Y.; Cheng, L.; Zhou, X. \& Ren, B. Transmission routes of $2019-n C o V$ and controls in dental practice. Int. J. Oral Sci., 12:9, 2020.

Sociedad Chilena de Cirugía y Traumatología Bucal y Maxilofacial. Covid-19: Recomendaciones de la Sociedad de Cirugía y Traumatología Bucal y Maxilofacial de Chile. Santiago de Chile, Sociedad Chilena de Cirugía y Traumatología Bucal y Maxilofacial, 2020. Disponible en: http://www.colegiodentistas.cl/inicio/2020/ 03/22/covid-19-recomendaciones-de-la-sociedad-de-cirugia-ytraumatologia-bucal-y-maxilofacial-de-chile/

Subsecretaría de Redes Asistenciales. Lineamientos para reducción de riesgo de transmisión y contagio en el retorno gradual de la atención odontológica en contexto pandemia COVID-19. Santiago de Chile, Subsecretaría de Redes Asistenciales, Ministerio de Salud, Gobierno de Chile, 2020. Disponible en: http:/ /www.colegiodentistas.cl/inicio/wp-content/uploads/2020/07/ ORD-2246-lineamientos-para-reduccion-de-riesgos-detransmision-y-contagio-en-el-retorno-gradual-de-la-atencionodontologica-en-contexto-pandemia-covid-19-21-07-2020.pdf

Subsecretaría de Salud Pública. Orientaciones para Atención Odontológica en Fase IV COVID-19. Santiago de Chile, Subsecretaría de Salud Pública, Ministerio de Salud, Gobierno de Chile, 2020a.

Subsecretaría de Salud Pública. Protocolo de Manejo de Contactos de Casos COVID-19, Fase 4. Versión 2. Santiago de Chile, Subsecretaría de Salud Pública, Ministerio de Salud, Gobierno de Chile, 2020b. Disponible en: https://www.uchile.cl/noticias/ 162461/protocolo-de-manejo-de-contactos-de-casos-covid-19fase-4

To, K. K.; Tsang, O. T. Y.; Yip, C. C. Y.; Chan, K. H.; Wu, T. C.; Chan, J. M. C.; Leung, W. S.; Chik, T. S. H.; Choi, C. Y. C.; Kandamby, D. H.; et al. Consistent detection of 2019 Novel Coronavirus in saliva. Clin. Infect. Dis., 71(15):841-3, 2020.

Vargas-Buratovic, J. P.; Verdugo-Paiva, F.; Véliz-Paiva, C.; LópezTagle, E.; Ahumada-Salinas, A. \& Ortuño-Borroto, D. Dental recommendations in the COVID-19 pandemic: A narrative review. Medwave, 20(5):e7916, 2020.

World Health Organization. WHO Coronavirus Disease (COVID-19) Dashboard. Ginebra, World Health Organization, 2020.

World Health Organization. WHO Guidelines on Hand Hygiene in Health Care First Global Patient Safety Challenge Clean Care is Safer Care. Ginebra, World Health Organization, 2009. Disponible en: https://apps.who.int/iris/bitstream/handle/10665/44102/ 9789241597906 eng.pdf;jsessionid=A80967E9D596029669E4 B0E2EF2128F3? sequence $=1$
Dirección para correspondencia:

Inés Aylwin Miranda

Cirujano Dentista

Pontificia Universidad Católica de Chile

CHILE

Email: ifaylwin@uc.cl 